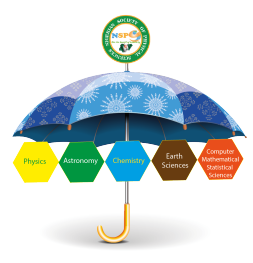

\title{
The Solution of a Mathematical Model for Dengue Fever Transmission Using Differential Transformation Method
}

\author{
Felix Yakubu Eguda*, Andrawus James, Sunday Babuba \\ Department of Mathematics, Federal University, Dutse, Jigawa State.
}

\begin{abstract}
Differential Transformation Method (DTM) is a very effective tool for solving linear and non-linear ordinary differential equations. This paper uses DTM to solve the mathematical model for the dynamics of Dengue fever in a population. The graphical profiles for human population are obtained using Maple software. The solution profiles give the long term behavior of Dengue fever model which shows that treatment plays a vital role in reducing the disease burden in a population.
\end{abstract}

Keywords: Dengue Fever, Mathematical Model, Differential Transformation Method, Ordinary Differential Equations

\section{Article History :}

Received: 15 June 2019

Received in revised form: 13 July 2019

Accepted for publication: 16 July 2019

Published: 03 September 2019

\section{Introduction}

Dengue fever is an infectious vector borne disease spreading in tropical and subtropical countries with more than 50 million dengue fever cases per year. It is transmitted to humans by the bite of infected aedes mosquitoes. The major vector, Aedes aegypti, thrives in tropical regions, mainly in urban areas, closely linked to human populations providing artificial water-holding containers as breeding sites. A second potential vector, Aedes Albopictus, resides in temperate regions (North America and Europe), where it may give rise to occasional dengue outbreaks. [1,2].

As part of awareness campaigns, different kinds of precautions have been suggested towards preventing mosquito's bite. Some of the precautions that can be taken are: to keep home, environment and surrounding clean, to remove all stagnant water and containers, to cover all containers properly to prevent

${ }^{*}$ Corresponding Author Tel. No: +2348160559365

Email address: felyak_e@yahoo.co.uk (Felix Yakubu Eguda ) dengue mosquito breeding there, to use mosquito repellents to avoid mosquito bite, to use mosquitoes net around bed while sleeping etc. [1]. Different studies have shown the importance of mathematical approaches in understanding dengue disease transmission and evaluating the effectiveness and/or costeffectiveness of control strategies [1].

In recent years, the field of public health has benefited tremendously from the use of mathematical models to study the spread of infectious disease. Most epidemiological models are represented using systems of non-linear ordinary differential equations [3]. Differential Transformation Method is a semi-analytical method of solving both linear and nonlinear system of ordinary differential equations (ODE) to obtain approximate series solutions. This method which was derived from the Taylor's series expansion has been used to solve problems in Mathematics and Physics [4], fractional differential- algebraic equations [5], fourth-order parabolic partial differential equations [6], fractional-order integrodifferential equations [7], differential equation [8] and problems in epidemic models [9]. 


\section{Materials and Methods}

\subsection{Model Formulation}

Two populations consisting of human and vector population will be considered in this work. The model sub-divides these populations into a number of mutually-exclusive compartments, as given below. The total population of human and vectors is divided into the following mutually exclusive epidemiological classes, namely, susceptible humans $\left(S_{H}(t)\right)$, humans with dengue in latent stage $\left(E_{1}(t)\right)$, humans with dengue ( $I_{1}(t)$ ), humans treated of dengue $\left(R_{1}(t)\right)$, susceptible vectors $\left(S_{V}(t)\right)$, vectors with latent dengue $\left(E_{V}(t)\right)$, vectors with dengue $\left(I_{V}(t)\right)$.

\subsection{Derivation of Model Equations}

Let $N_{H}(t)$ and $N_{V}(t)$ denote the total number of humans and vectors at time $t$, respectively. Hence, we have that,

$$
N_{H}(t)=S_{H}(t)+E_{1}(t)+I_{1}(t)+R_{1}(t)
$$

and

$$
N_{V}(t)=S_{V}(t)+E_{V}(t)+I_{V}(t)
$$

Susceptible humans are recruited at a rate ${ }_{H}$ while the susceptible vectors are recruited at a rate $V$.

Susceptible humans contract dengue at a rate

$$
\lambda_{D V}=\frac{\beta_{V H}\left(\eta_{v} E_{v}+I_{v}\right)}{N_{H}},
$$

where $\eta_{v}<1$, this accounts for the relative infectiousness of vectors with latent dengue $I_{v}$ compared to vectors in the $I_{v}$ class.

Susceptible vectors acquire dengue infection from infected humans at a rate

$$
\lambda_{D H}=\frac{\beta_{H V}\left(\eta_{A} E_{1}+\eta_{B} I_{1}\right)}{N_{H}},
$$

where $\eta_{A}<\eta_{B}$ this accounts for the relative infectiousness of humans with latent dengue $E_{1}$ compared to humans in the $I_{1}$ class [10].

The model equations for dengue disease transmission incorporating treatment as a control measure are given below:

$$
\begin{aligned}
& S_{H}=\Lambda_{H}-\mu_{H} S_{H}-\lambda_{D V} S_{H} \\
& E_{1}=\lambda_{D V} S_{H}-\left(\gamma_{1}+\mu_{H}\right) E_{1} \\
& I_{1}=\gamma_{1} E_{1}-\left(\tau_{1}+\mu_{H}+\delta_{D 1}\right) I_{1} \\
& R_{1}=\tau_{1} I_{1}-\mu_{H} R_{1} \\
& S_{V}=\Lambda_{V}-\mu_{V} S_{V}-\lambda_{D H} S_{V} \\
& E_{V}=\lambda_{D H} S_{V}-\left(\gamma_{V}+\mu_{V}\right) E_{V} \\
& I_{V}=\gamma_{V} E_{V}-\left(\mu_{V}+\delta_{H V}\right) I_{V}
\end{aligned}
$$

\subsection{Differential Transformation Method (DTM)}

An arbitrary function $f(t)$ can be expanded in Taylor series about a point $t=0$ as

$$
f(t)=\sum_{k=0}^{\infty} \frac{t^{k}}{k !}\left[\frac{d^{k} f}{d t^{k}}\right]_{t=0}
$$

The differential transformation of $f(t)$ is defined as

$$
F(t)=\frac{1}{k !}\left[\frac{d^{k} f}{d t^{k}}\right]_{t=0}
$$

Then the inverse differential transform is

$$
f(t)=\sum_{k=0}^{\infty} t^{k} F(t)
$$

If $y(t)$ and $g(t)$ are two uncorrelated functions with $t$ where $Y(k)$ and $G(k)$ are the transformed functions corresponding to $y(t)$ and $g(t)$ then, the fundamental mathematical operations performed by differential transform can be proved easily and are listed as follows

Table 1: The Fundamental Mathematical Operations by Differential Transformation Method (DTM). Source: [11, 12]

\begin{tabular}{lr}
\hline Transformed Function & Original Function \\
\hline$y(t)=f(t) \pm g(t)$ & $Y(k)=F(k) \pm G(k)$ \\
$y(t)=a f(t)$ & $Y(k)=a F(k)$ \\
$y(t)=\frac{d f(t)}{d t}$ & $Y(k)=(k+1) F(k+1)$ \\
$y(t)=\frac{d^{2} f(t)}{d t^{2}(t)}$ & $Y(k)=(k+1)(k+2) F(k+2)$ \\
$y(t)=\frac{d^{t} f(t)}{d t^{m}}$ & $Y(k)=(k+1)(k+2) \ldots(k+m) F(k+m)$ \\
$y(t)=1$ & $Y(k)=\delta(k)$ \\
$y(t)=t$ & $Y(k)=\delta(k-1)$ \\
$y(t)=t^{m}$ & $Y(k)=\delta(k-m)=\left\{\begin{array}{l}1, k=m \\
0, k \neq m\end{array}\right.$ \\
$y(t)=f(t) g(t)$ & $Y(k)=\sum_{m=0}^{k} G(m) f(k-m)$ \\
$y(t)=e^{(\lambda t)}$ & $Y(k)=\frac{\lambda^{k}}{k !}$ \\
$y(t)=(1+t)^{m}$ & $Y(k)=\frac{(m(m-1) \ldots(m-k+1))}{k !}$
\end{tabular}

\subsection{Analytical solution of the model equations using differen- tial transformation method (DTM)}

In this section, the Differential Transformation Method (DTM) is employed to solve the system of non-linear differential equations which describe our model for Dengue fever.

Let the model equation be a function $q(t), q(t)$ can be expanded in Taylor series about a point $t=0$ as

$$
q(t)=\sum_{k=0}^{\infty} \frac{t^{k}}{k !}\left[\frac{d^{k} q}{d t^{k}}\right]_{t=0},
$$

where,

$$
q(t)=\left\{s_{H}(t), E_{1}(t), I_{1}(t), R_{1}(t), S_{v}(t), E_{v}(t), I_{v}(t)\right\}(5)
$$

The differential transformation of $q(t)$ is defined as

$$
Q(t)=\frac{1}{k !}\left[\frac{d^{k} q}{d t^{k}}\right]_{t=0}
$$


Table 2: Values for parameters used for analytical solutions

\begin{tabular}{|c|c|c|c|c|}
\hline Parameter & Description & Values & Unit & Reference \\
\hline$\Lambda_{H}, \Lambda_{V}$ & $\begin{array}{l}\text { Recruitment rate into the population of susceptible hu- } \\
\text { mans and vectors respectively. }\end{array}$ & 500,10000000 & Year $^{-1}$ & [14] \\
\hline$\mu_{H}, \mu_{v}$ & Natural death for humans, vectors respectively. & $0.02041,0.5$ & Year $^{-1}$ & [13] \\
\hline$\beta_{V H}$ & $\begin{array}{l}\text { Effective contact rate for } 0.5 \text { dengue from vectors to } \\
\text { humans }\end{array}$ & 0.5 & Year $^{-1}$ & [14] \\
\hline$\beta_{H V}$ & $\begin{array}{l}\text { Effective contact rate for } 0.4 \text { dengue from humans to } \\
\text { vectors }\end{array}$ & 0.4 & Year $^{-1}$ & [14] \\
\hline$\tau_{1}$ & Dengue treatment rate for $I_{1}$ & $(0,1)$ & Ind $^{-1}$ Year $^{-1}$ & {$[14]$} \\
\hline$\gamma_{1}$ & Progression rate to active dengue & 0.3254 & Year $^{-1}$ & [14] \\
\hline$\gamma_{V}$ & Progression rate to active dengue (vectors) & 0.03 & Year $^{-1}$ & [14] \\
\hline$\delta_{D_{1}}$ & Disease induced death Dengue & 0.365 & Year $^{-1}$ & [13] \\
\hline$\delta_{H V}$ & Disease induced death dengue (vectors) & 0 & Year $^{-1}$ & {$[14]$} \\
\hline$\eta_{V}, \eta_{A}, \eta_{B}$ & Modification parameters for $E_{v}, E_{1}, i_{1}$ & $0.4,1.2,0.5$ & Year $^{-1}$ & [13] \\
\hline
\end{tabular}

Then the inverse differential transform is

$$
q(t)=\sum_{k=0}^{\infty} t^{k} Q(t) .
$$

Using the fundamental operations of differential transformation method in Table 1, we obtain the following recurrence relation of equation (1) as

$$
\begin{aligned}
& S_{H}(k+1)= \frac{1}{k+1}\left[\Lambda_{H}-\mu_{H} S_{H}(k)\right. \\
&-\frac{\beta_{V H} \eta_{v}}{N_{H}} \sum_{m=0}^{k} S_{H}(m) E_{V}(k-m) \\
&\left.-\frac{\beta_{V H}}{N_{H}} \sum_{m=0}^{k} S_{H}(m) I_{V}(k-m)\right] \\
& E_{1}(k+1)= \frac{1}{k+1}\left[\frac{\beta_{V H} \eta_{v}}{N_{H}} \sum_{m=0}^{k} S_{H}(m) E_{V}(k-m)\right. \\
&-\frac{\beta_{V H}}{N_{H}} \sum_{m=0}^{k} S_{H}(m) I_{V}(k-m) \\
&\left.-\left(\gamma_{1}+\mu_{H}\right) E_{1}(k)\right] \\
& I_{1}(k+1)=\frac{1}{k+1}\left[\gamma_{1} E_{1}(k)-\left(\tau_{1}+\mu_{H}+\delta_{D 1}\right) I_{1}\right]
\end{aligned}
$$

$$
\begin{aligned}
& E_{V}(k+1)= \frac{1}{k+1}\left[\frac{\beta_{H V} \eta_{A}}{N_{H}} \sum_{m=0}^{k} S_{V}(m) E_{1}(k-m)\right. \\
&-\frac{\beta_{H V} \eta_{B}}{N_{H}} \sum_{m=0}^{k} S_{V}(m) I_{1}(k-m) \\
&\left.-\left(\gamma_{v}+\mu_{v}\right) E_{V}(k)\right] \\
& I_{V}(k+1)=\frac{1}{k+1}\left[\gamma_{V} E_{V}(k)-\left(\mu_{V}+\delta_{H V}\right) I_{V}(k)\right]
\end{aligned}
$$

With the initial conditions

$$
\begin{aligned}
& S_{H}(0)=3503, E_{1}(0)=490, I_{1}(0)=390, R_{1}(0)=87, \\
& S_{V}(0)=390, E_{V}(0)=100, I_{V}(0)=190
\end{aligned}
$$

The parameter values are

$$
\begin{aligned}
& N_{H}=4470, N_{V}=610, \Lambda_{H}=500, \Lambda_{V}=1,000,000, \\
& \mu_{H}=0.02041, \mu_{V}=0.5, \beta_{V H}=0.5, \beta_{H V}=0.4, \\
& \tau_{1}=0.75, \gamma_{1}=0.3254, \gamma_{V}=0.03, \delta_{D 1}=0.365, \\
& \delta_{H V}=0, \eta_{V}=0.4, \eta_{A}=1.2, \eta_{B}=0.5
\end{aligned}
$$

We consider $k=0,1,2,3$. Cases $\mathrm{A} 1$ to $\mathrm{A} 3$ are the variation of different values of $\tau_{1}$

Case A1: High Dengue Treatment Rate, $\tau_{1}=0.75$

$$
\begin{array}{r}
S_{H}(1)=-237.6147983, S_{H}(2)=12320.67062, \\
S_{H}(3)=-146425.8636, S_{H}(4)=1347694.18, \\
E_{1}(1)=-522.0979067, E_{1}(2)=6637.369770, \\
E_{1}(3)=-82219.53607, E_{1}(4)=775491.4995, \\
I_{1}(1)=-283.36390, I_{1}(2)=75.92177345, \\
I_{1}(3)=691.1992607, I_{1}(4)=-6884.757898, \\
R_{1}(1)=290.72433, R_{1}(2)=-109.2283043, \\
R_{1}(3)=19.72355993, R_{1}(4)=129.4992219, \\
S_{V}(1)=-14008.26174, S_{V}(2)=260942.4174,
\end{array}
$$




$$
\begin{array}{r}
S_{V}(3)=-3239718.545, S_{V}(4)=30208553.32, \\
E_{V}(1)=-3515.845638, E_{V}(2)=61669.12070, \\
E_{V}(3)=-717387.0983, E_{V}(4)=6204920.468, \\
I_{V}(1)=-4742.00, I_{V}(2)=86488.76230, \\
I_{V}(3)=-1051663.250, I_{V}(4)=9591046.752 .
\end{array}
$$

Then the closed form of the solution where $k=0,1,2,3$ can be written as

$$
\begin{aligned}
S_{H}(t)= & \sum_{k=0}^{\infty} S_{H}(k) t^{k}=3503-237.6147983 t \\
& +12320.67062 t^{2}-146425.8636 t^{3} \\
& +1347694.180 t^{4} \\
E_{1}(t)= & \sum_{k=0}^{\infty} E_{1}(k) t^{k}=490-522.0979067 t \\
& +6637.369770 t^{2}-82219.53607 t^{3} \\
& +775491.4995 t^{4} \\
I_{1}(t)= & \sum_{k=0}^{\infty} I_{1}(k) t^{k}=390-283.36390 t \\
& +75.92177345 t^{2}+691.1992607 t^{3} \\
& -6884.757898 t^{4} \\
R_{1}(t)= & \sum_{k=0}^{\infty} R_{1}(k) t^{k}=87+290.72433 t \\
& -109.2283043 t^{2}+19.72355993 t^{3} \\
& +129.4992219 t^{4} \\
& +9591046.752 t^{4} \\
& \sum_{k=0}^{\infty} S_{V}(k) t^{k}=390-14008.26174 t \\
& +260942.4174 t^{2}-3239718.545 t^{3} \\
& +30208553.32 t^{4} \\
S_{V}(t)= & \sum_{k=0}^{\infty} E_{V}(k) t^{k}=100-3515.845638 t \\
& +61669.12070 t^{2}-717387.0983 t^{3} \\
I_{V}(t)= & \sum_{k=0}^{\infty} I_{V}(k) t^{k}=130-4742.00 t \\
E_{V}(t)= & 86488.76230 t^{2}-1051663.250 t^{3} \\
& +620.468 t^{4} \\
& +150
\end{aligned}
$$

Case A2: Moderate Dengue Treatment Rate, $\tau_{1}=0.5$

$S_{H}(1)=-237.6147983, S_{H}(2)=12320.67062$,

$S_{H}(3)=-146421.4193, S_{H}(4)=1347571.999$,

$E_{1}(1)=-522.0979067, E_{1}(2)=6637.369770$,

$E_{1}(3)=-82223.98033, E_{1}(4)=775613.8858$,

$I_{1}(1)=-185.86390, I_{1}(2)=-2.662451550$,

$I_{1}(3)=720.7191613, I_{1}(4)=-6848.453788$,

$R_{1}(1)=193.22433, R_{1}(2)=-48.43782929$,

$R_{1}(3)=-0.1142032264, R_{1}(4)=90.09047788$
$S_{V}(1)=-14008.26174, S_{V}(2)=260933.9106$,

$S_{V}(3)=-3239404.789, S_{V}(4)=30202664.15$,

$E_{V}(1)=-3515.845638, E_{V}(2)=61660.61400$,

$E_{V}(3)=-71707.62417, E_{V}(4)=619913.9432$,

$I_{V}(1)=-4742.00, I_{V}(2)=86488.76230$,

$I_{V}(3)=-1051663.335, I_{V}(4)=9591049.860$. (19)

Then the closed form of the solution where $k=0,1,2,3$ can be written as

$$
\begin{aligned}
S_{H}(t)= & \sum_{k=0}^{\infty} S_{H}(k) t^{k}=3503-237.6147983 t \\
& +12320.67062 t^{2}-146421.4193 t^{3} \\
& +1347571.999 t^{4} \\
E_{1}(t)= & \sum_{k=0}^{\infty} E_{1}(k) t^{k}=490-522.0979067 t \\
& +6637.369770 t^{2}-82223.98033 t^{3} \\
& +775613.8858 t^{4} \\
I_{1}(t)= & \sum_{k=0}^{\infty} I_{1}(k) t^{k}=390-185.86390 t \\
& -2.662451550 t^{2}+720.7191613 t^{3} \\
& -6848.453788 t^{4} \\
R_{1}(t)= & \sum_{k=0}^{\infty} R_{1}(k) t^{k}=87+193.22433 t \\
& -48.43782929 t^{2}-0.1142032264 t^{3} \\
& +90.09047788 t^{4} \\
& \sum_{k=0}^{\infty} S_{V}(k) t^{k}=390-14008.26174 t \\
& +260933.9106 t^{2}-3239404.789 t^{3} \\
& +30202664.15 t^{4} \\
S_{V}(t)= & \sum_{k=0}^{\infty} E_{V}(k) t^{k}=100-3515.845638 t \\
& +61660.61400 t^{2}-717076.2417 t^{3} \\
& +6199139.432 t^{4} \\
I_{V}(t)= & \sum_{k=0}^{\infty} I_{V}(k) t^{k}=130-4742.00 t \\
& +86488.76230 t^{2}-1051663.335 t^{3} \\
E_{V}(t)= & +9591049.860 t^{4} \\
= &
\end{aligned}
$$

Case A3: Low Dengue Treatment Rate, $\tau_{1}=0.25$

$$
\begin{aligned}
& S_{H}(1)=-237.6147983, S_{H}(2)=12320.67062, \\
& S_{H}(3)=-1464169750, S_{H}(4)=1347571.999, \\
& E_{1}(1)=-522.0979067, E_{1}(2)=6637.369770, \\
& E_{1}(3)=-82223.98033, E_{1}(4)=775613.8858, \\
& I_{1}(1)=-88.36390, I_{1}(2)=-56.87167655, \\
& I_{1}(3)=720.7191613, I_{1}(4)=-6848.453788, \\
& R_{1}(1)=95.72433, R_{1}(2)=-12.02235429,
\end{aligned}
$$




$$
\begin{aligned}
& R_{1}(3)=-0.1142032264, R_{1}(4)=90.09047788 \\
& S_{V}(1)=-14008.26174, S_{V}(2)=260925.4039, \\
& S_{V}(3)=-3239404.789, S_{V}(4)=30202664.15, \\
& E_{V}(1)=-3515.845638, E_{V}(2)=61652.10730, \\
& E_{V}(3)=-71707.62417, E_{V}(4)=619913.9432, \\
& I_{V}(1)=-4742.00, I_{V}(2)=86488.76230, \\
& I_{V}(3)=-1051663.335, I_{V}(4)=9591049.860 .
\end{aligned}
$$

Then the closed form of the solution where $k=0,1,2,3$ can be written as

$$
\begin{aligned}
S_{H}(t)= & \sum_{k=0}^{\infty} S_{H}(k) t^{k}=3503-237.6147983 t \\
& +12320.67062 t^{2}-146416.9750 t^{3} \\
& +1347450.373 t^{4} \\
E_{1}(t)= & \sum_{k=0}^{\infty} E_{1}(k) t^{k}=490-522.0979067 t \\
& +6637.369770 t^{2}-82228.42463 t^{3} \\
& +775735.7158 t^{4}, \\
I_{1}(t)= & \sum_{k=0}^{\infty} I_{1}(k) t^{k}=390-88.36390 t \\
& -56.87167655 t^{2}+731.9789850 t^{3} \\
& -6805.559035 t^{4}, \\
R_{1}(t)= & \sum_{k=0}^{\infty} R_{1}(k) t^{k}=87+95.72433 t \\
& -12.02235429 t^{2}-4.657514297 t^{3} \\
& +45.77245152 t^{4}, \\
& +9591052.958 t^{4} . \\
& \sum_{k=0}^{\infty} S_{V}(k) t^{k}=390-14008.26174 t \\
& +260925.4039 t^{2}-323909.2451 t^{3} \\
& +30196827.38 t^{4}, \\
S_{V}(t)= & \sum_{k=0}^{\infty} I_{V}(k) t^{k}=130-4742.00 t \\
E_{V}(t)= & \sum_{k=0}^{\infty} E_{V}(k) t^{k}=100-3515.845638 t \\
& +61652.10730 t^{2}-717666.8033 t^{3} \\
& +86488.76230 t^{2}-1051663.420 t^{3} \\
& \\
&
\end{aligned}
$$

\subsection{Numerical Simulation and Graphical Representation of the} Solutions of the Model Equations

The numerical simulation which illustrates the analytical solution of the Model is demonstrated using Maple software. This is achieved by using some set of parameter values given in the Table 2. The following initial conditions for the human populations $S_{H}(0)=3503, E_{1}(0)=490, I_{1}(0)=390$, $R_{1}(0)=87, S_{V}(0)=390, E_{V}(0)=100, I_{V}(0)=190$ are considered.

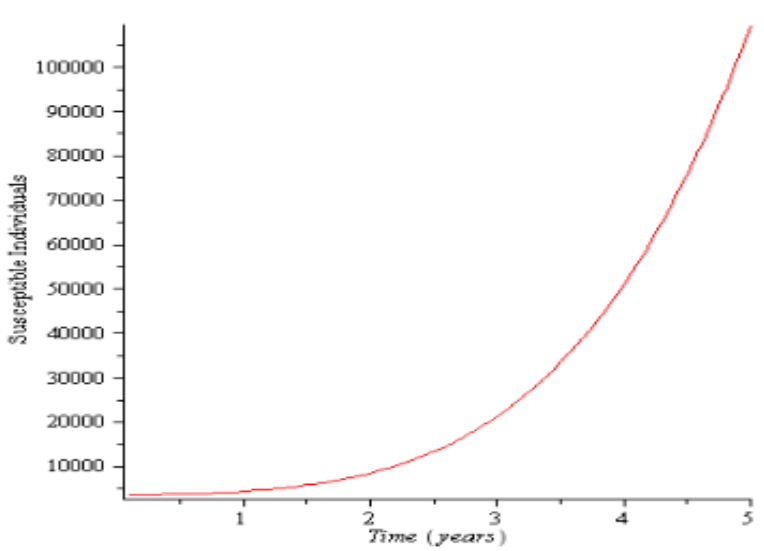

Figure 1: Solution of Susceptible Population Using DTM

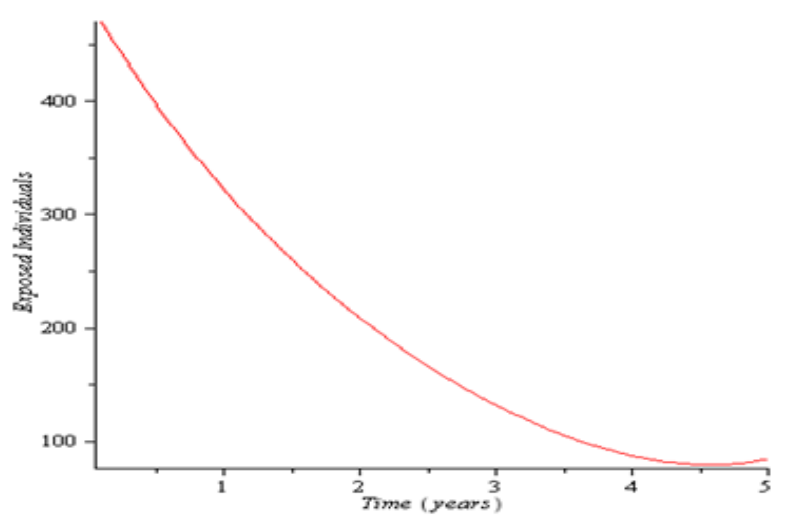

Figure 2: Solution of Exposed Population Using DTM

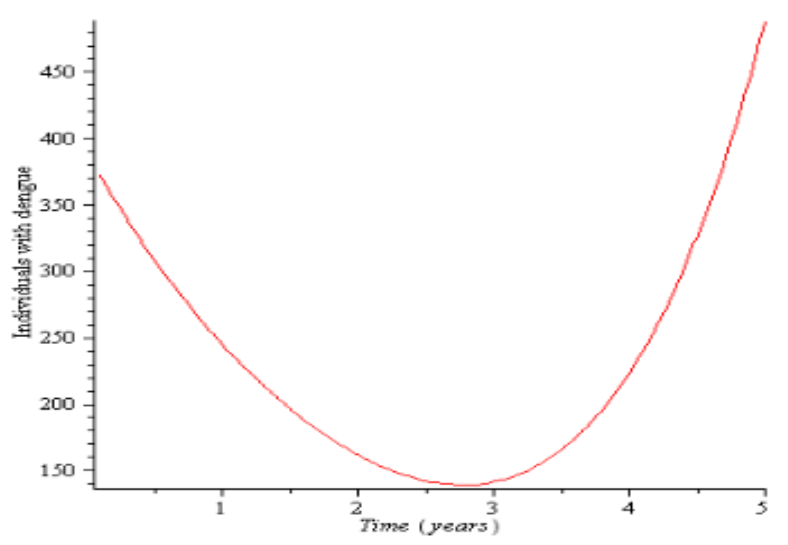

Figure 3: Solution of Human Population with Dengue Using DTM

\section{Discussion of results}

The Figures 1 to 6 give the numerical profiles of the solutions (17), (19) and (21) using DTM. Figure 1 shows increase in the population of susceptible individuals while Figure 2 indicates a decreasing population of the exposed owing to the progression out of exposed class to class of human with Dengue. Figure 3 implies a decrease in the population of human infected with dengue which later increases. Figure 4 implies the treated 


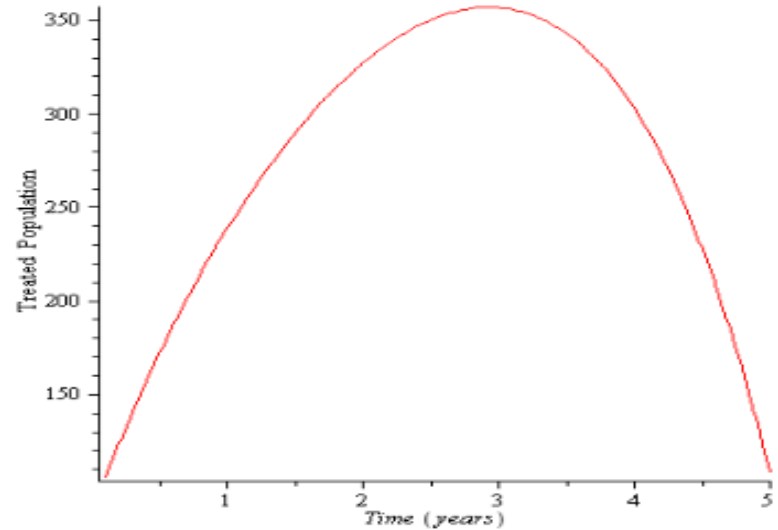

Figure 4: Solution of Treated Human Population Using DTM

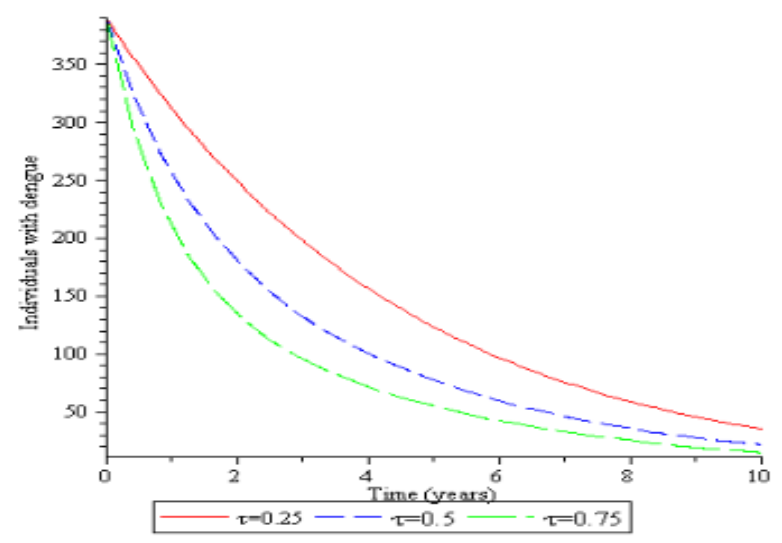

Figure 5: The Effect of Different Treatment Rates on Human Population with Dengue Using DTM

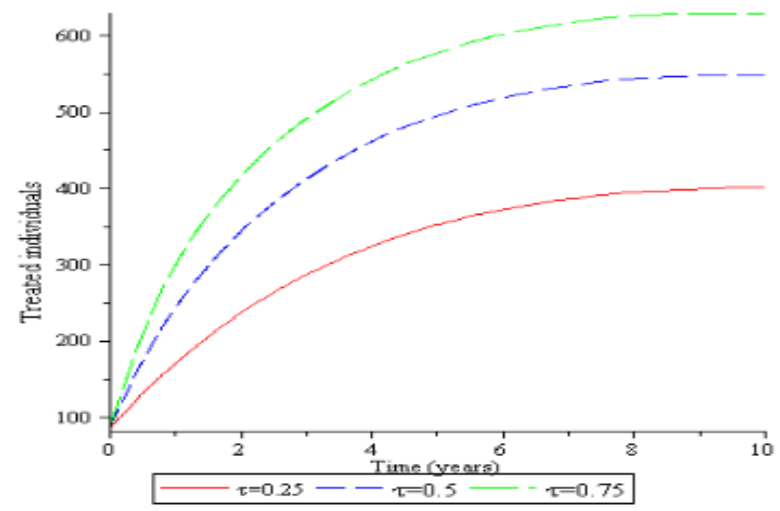

Figure 6: The Effect of Different Treatment Rates on Treated Human Population Using DTM

human population increases sharply to a point and then decreases. Figure 5 indicates that increasing the treatment rate of individuals in the infected population leads to a reduction in the number of infected individuals which is due to progression into the treated population while Figure 6 shows that increasing the treatment rate of individuals in the infected population leads to a corresponding increase in the treated population.

\section{Conclusion}

We formulated a compartmental model to investigate the dynamics of dengue fever in a population with treatment as a control measure. Differential Transform Method (DTM) was employed to obtain the series solution of the model. Numerical simulations were carried out to determine the long term behavior of Dengue fever model which shows that treatment plays a vital role in reducing the disease burden in a population. The results of the simulations were displayed graphically.

\section{Acknowledgments}

We thank the referees for the positive enlightening comments and suggestions, which have greatly helped us in making improvements to this paper.

\section{References}

[1] G. R. Phaijoo, \& D. B. Gurung, "Mathematical Model of Dengue Fever with and without awareness in Host Population", International Journal of Advanced Engineering Research and Applications 1 (2015) 2454.

[2] M. Andraud, N. Hens, C. Marais \& P. Beutels, "Dynamic Epidemiological Models for Dengue Transmission: A systematic Review of Structural Approaches", PLOS ONE 7 (2012) 1.

[3] S. A. Carvalho, S. O. Silva, \& C.I.Charret "Mathematical Modeling of Dengue Epidemic: Control Methods and Vaccination Strategies", Theory Biosci. (2019). https://doi.org/10.1007/s12064-019-00273-7.

[4] B. Ibis, M.Bayram \& G. Agargun, "Applications of Fractional Differential Transform Method to Fractional Differential-Algebraic Equations", European Journal of Pure and Applied Mathematics 4 (2011) 5543.

[5] M. A. E. Osman, K. K.Adu \& C.Yang, "A Simple SEIR Mathematical Model of Malaria Transmission", Asian Research Journal of Mathematics 7 (2017) 1.

[6] D. Nazari \& S. Shahmorad "Application of the fractional differential transform method to fractional-order integro-differential equations with nonlocal boundary conditions", Journal of Computational and Applied Mathematics, 234 (2010) 883

[7] G. Methi, "Solution of Differential Equation Using Differential Transform Method" , Asian Journal of Mathematics \& Statistics 9 (2016) 13.

[8] F. Mirzaee, "Differential Transform Method for Solving Linear and Nonlinear Systems of Ordinary Differential Equations", Applied Mathematical Sciences 5 (2011) 70.

[9] A. A. Idowu, I. M. Olanrewaju, P. O. James, S .Amadiegwu \& F.A.Oguntolu, "Differential Transform Method for Solving Mathematical Model of SEIR and SEI Spread of Malaria", International Journal of Sciences: Basic and Applied Research (IJSBAR), 40 (2018) 197.

[10] J. Andrawus \& F. Y. Eguda, "Analysis of a Mathematical Model to Investigate the Dynamics of Dengue Fever" J. Appl. Sci. Environ. Manage. 21 (2017) 626

[11] O. J. Peter \& M. O. Ibrahim,"Application of Differential Transform Method in Solving a Typhoid Fever Model", International Journal of Mathematical Analysis and Optimization: Theory and Applications. (2017) 250.

[12] S. A. Somma, N. I. Akinwande, R. A. Abah, F. A. Oguntolu \& F.D.Ayegbusi ,"Semi-analytical Solution for the Mathematical Modeling of yellow fever dynamics incorporating secondary host", Communication in Mathematical Modeling and Applications 4 (2019) 9.

[13] D. Okuonghae \& S. E. Omosigho, "Analysis of Mathematical Model for Tuberculosis", Journal of Theo. Biol. 269 (2011) 31.

[14] S. M. Garba, A. B. Gumel \& M.R.Abubakar, "Backward Bifurcations in Dengue Transmission Dynamics”, Mathematical Biosciences 215 (2008) 11. 